TRANSACTIONS OF THE

AMERICAN MATHEMATICAL SOCIETY

Volume 281. Number 1, January 1984

\title{
ADDITIVITY OF MEASURE IMPLIES ADDITIVITY OF CATEGORY
}

BY

TOMEK BARTOSZYŃSKI

\begin{abstract}
In this paper it is proved that $2^{\omega}$-additivity of category follows from $2^{\omega}$-additivity of measure, and a combinatorial characterization of additivity of measure is found.
\end{abstract}

Definitions. For abbreviation we denote by $A(c), B(c), A(m), B(m), D$ and $h D$ the following sentences:

$A(c) \equiv$ union of less than $2^{\omega}$ meager sets is meager.

$B(c) \equiv$ union of less than $2^{\omega}$ meager sets is not $\omega^{\omega}$.

$A(m)$ and $B(m)$ are defined analogously by replacing the word "meager" by "Lebesgue measure zero". The symbols $\forall^{\infty}, \exists^{\infty}$ abbreviate "for all but finitely many" and "exist infinitely many". Let $\prec$ denote the following order on $\omega^{\omega}$. For $f, g \in \omega^{\omega}, f \prec g \equiv \forall^{\infty} n f(n)<g(n)$.

$D \equiv \forall F \subset \omega^{\omega}\left[|F|<2^{\omega} \rightarrow \exists g \in \omega^{\omega} \forall f \in F f \prec g\right]$.

$h D \equiv$ for every family of power less than $2^{\omega}$ which consists of converging series there exists a converging series eventually dominating each of them.

Recall here some known facts which will be used later.

Theorem 1 (Miller, Truss). $A(c) \equiv B(c) \& D$.

Theorem 2 (Miller). Assuming $D$

$$
B(c) \equiv \forall F \subset \omega^{\omega}\left[|F|<2^{\omega} \rightarrow \exists g \in \omega^{\omega} \forall f \in F \exists^{\infty} n f(n)=g(n)\right] .
$$

TheOREM 3 (Kunen). $A(c) \nrightarrow A(m)$.

For the proofs see [1].

TheOREM 4 (Miller). $A(m) \rightarrow D$.

Corollary (Miller). $A(m) \& B(c) \rightarrow A(c)$.

For the proof of Theorem 4 see [2].

Now we will find a combinatorial characterization of $A(m)$ which will be useful to establish the main result.

THEOREM 5. $A(m) \equiv h D$.

Received by the editors December 26, 1982.

1980 Mathematics Subject Classification. Primary 03E05; Secondary 28A99.

1984 American Mathematical Society $0002-9947 / 84 \$ 1.00+\$ .25$ per page 
Proof. $\leftarrow$ We will work in the space $R$ with Lebesgue measure $m$. Let $\left\{I_{n}: n<\omega\right\}$ be the enumeration of the standard basis of $R$, i.e. open intervals with rational endpoints. Take any family $\left\{G_{\xi}: \xi<\lambda<2^{\omega}\right\}$ of null sets in $R$. For every $\xi<\lambda$ we can find basic intervals $\left\{I_{n}^{\xi}: n<\omega\right\}$ such that

$$
\forall_{\xi<\lambda} G_{\xi} \subset \bigcap \bigcup_{n>n} I_{m}^{\xi} \text { and } \forall_{\xi<\lambda} \sum_{n=1}^{\infty} m\left(I_{n}^{\xi}\right)<\infty .
$$

Now for $\xi<\lambda$ define $f_{\xi} \in 2^{\omega}$ as

$$
f_{\xi}(n)=\left\{\begin{array}{ll}
1 & I_{n}=I_{k}^{\xi} \text { for some } k \in \omega \\
0 & \text { in the other cases }
\end{array} \text { for } n<\omega .\right.
$$

By this definition we have

$$
\forall_{\xi<\lambda} \sum_{n=1}^{\infty} f_{\xi}(n) \cdot m\left(I_{n}\right)<\infty .
$$

Now take the family $\left\{\sum_{n=1} f_{\xi}(n) \cdot m\left(I_{n}\right): \xi<\lambda\right\}$. It is easy to see that using $h D$ we can get $f \in 2^{\omega}$ such that $\sum_{n=1}^{\infty} f(n) \cdot m\left(I_{n}\right)<\infty$ and $\forall_{\xi<\lambda} \forall^{\infty} n f_{\xi}(n) \leqslant f(n)$. Let $G=\bigcap_{n} \cup_{m>n, f(m)=1} I_{m}$. We have

$$
G_{\xi} \subset \bigcap_{n} \bigcup_{m>n} I_{n}^{\xi} \subset G \text { for } \xi<\lambda .
$$

This finishes the proof because $G$ is a null set.

$\rightarrow$ LEMMA. The following conditions are equivalent:

(i) $h D$.

(ii) $\forall F \subset \omega^{\omega}\left[|F|<2^{\omega} \rightarrow \exists I_{n} \subset \omega\left[\left|I_{n}\right|<n^{2} \wedge \forall f \in F \forall^{\infty} n f(n) \in I_{n}\right]\right]$.

(iii)

$$
\begin{aligned}
D \& \forall F \subset \omega^{\omega}\left[\left(|F|<2^{\omega} \& F \prec f \& \sum_{n=1}^{\infty} \frac{1}{f(n)}<\infty\right)\right. \\
\left.\quad \rightarrow \exists I_{n} \subset \omega \sum_{n=1}^{\infty} \frac{\left|I_{n}\right|}{f(n)}<\infty \forall g \in F \forall^{\infty} n g(n) \in I_{n}\right] .
\end{aligned}
$$

Proof of the lemma. (i) $\rightarrow$ (ii). Let $F=\left\{f_{\xi}: \xi<\lambda<2^{\omega}\right\} \subset \omega^{\omega}$. Define for $\xi<\lambda$ the series

$$
a_{n}^{\xi}= \begin{cases}\max \left\{k^{-2}: n=f_{\xi}(k)\right\}, & n \in \operatorname{Rg}\left(f_{\xi}\right), \\ 0, & n \notin \operatorname{Rg}\left(f_{\xi}\right) .\end{cases}
$$

By $h D$ there exists $\sum_{n=1}^{\infty} a_{n}$ eventually dominating all $\sum_{n=1}^{\infty} a_{n}^{\xi}, \sum_{n=1}^{\infty} a_{n}<1$. Denote $I_{k}=\left\{n: a_{n} \geqslant k^{-2}\right\}$ for $k<\omega$. Notice that $\left|I_{n}\right|<n^{2}$ for almost every $n<\omega$ and $\forall g \in F \forall \forall^{\infty} n g(n) \in I_{n}$. Q.E.D.

(ii) $\rightarrow$ (i). Take any family of coverging series, i.e. $F=\left\{f_{\xi}: \xi<\lambda<2^{\omega}\right\}$ where $f_{\xi}: \omega \rightarrow Q$ (rationals) for every $\xi<\lambda$. Define for $\xi<\lambda$ sequences $\left\{n_{k}^{\xi}: k<\omega\right\} \subset \omega$ 
such that

$$
\forall \forall_{\xi<\lambda} \forall^{\infty} k \sum_{i>n_{k}^{\xi}}^{\infty} f_{\xi}(i)<2^{-k}
$$

Notice that (ii) obviously implies $D$, so we can find $h \in \omega^{\omega}$ such that

$$
\forall_{\xi<\lambda} \forall^{\infty} k n_{k}^{\xi}<h(k) \text {. }
$$

Now define

$$
f_{\xi}^{\prime}(k)=f_{\xi \mid[h(k), h(k+1))} \text { for } \xi<\lambda .
$$

We apply (ii) to the family $\left\{f_{\xi}^{\prime}: \xi<\lambda\right\}$ to get $\left\{I_{n}: n<\omega\right\}$ such that $\forall k\left|I_{k}\right|<k^{2}$ and $\forall_{\xi<\lambda} \forall \forall^{\infty} k f_{\xi}^{\prime}(k) \in I_{k}$. Each $I_{k}$ consists of functions from $[h(k), h(k+1))$ to $Q$. Now define a series $f: \omega \rightarrow Q$ in the following way:

$$
f(n)=\sup \left\{s(n): s \in I_{k} \text { and } \sum_{i=h(k)}^{h(k+1)} s(i)<2^{-k}\right\} .
$$

for $n \in[h(k), h(k+1))$. Notice that $\sum_{n=1}^{\infty} f(n) \leqslant \sum_{n=1}^{\infty} n^{2} / 2^{n}<\infty$ and $\forall_{\xi<\lambda} \forall^{\infty} n$ $f_{\xi}(n) \leqslant f(n)$, so $f$ is what we were looking for.

(ii) $\rightarrow$ (iii). Take any family $F \subset \omega^{\omega}$ such that $|F|<2^{\omega}, F \prec f$ and $\sum_{n=1}^{\infty} 1 / f(n)<$ $\infty$. We have to find $\left\{I_{n}: n<\omega\right\}$ such that

$$
\sum_{n=1}^{\infty} \frac{\left|I_{n}\right|}{f(n)}<\infty \quad \text { and } \quad \forall g \in F \forall^{\infty} n g(n) \in I_{n} .
$$

Let $\left\{R_{n}: n<\omega\right\}$ be a nondecreasing sequence such that

$$
\sum_{n=1}^{\infty} \frac{R_{n}}{f(n)}<\infty \text { and } R_{n} \underset{n \rightarrow \infty}{\rightarrow} \infty .
$$

Pick a sequence $\left\{u_{n}: n<\omega\right\}$ such that $\forall n R_{u_{n}} \geqslant n^{2}$. For $g \in F$ let $g^{\prime}$ be defined in the following way:

$$
g^{\prime}(k)=g_{\left.\| u_{k}, u_{k+1}\right)} \text { for } k<\omega .
$$

Applying (ii) to the family $\left\{g^{\prime}: g \in F\right\}$ and define as in the previous proof:

$$
J_{n}=\left\{s(n): s \in I_{k}\right\} \quad \text { for } n \in\left[u_{k}, u_{k+1}\right) .
$$

It is easy to see that

$$
\forall g \in F \forall^{\infty} n \quad g(n) \in J_{n} \quad \text { and } \quad \forall^{\infty} n \quad\left|J_{n}\right| \leqslant R_{n} .
$$

As we noticed earlier (ii) $\rightarrow D$ so the implication (ii) $\rightarrow$ (iii) is proved.

(iii) $\rightarrow$ (ii). Take any $F \subset \omega^{\omega},|F|<2^{\omega}$. By $D$ we can find $f \in \omega^{\omega}$ and a strictly increasing sequence $\left\{k_{n}: n<\omega\right\} \subset \omega$ such that $F \prec f$ and $k_{n} / f(n)=n^{-2}$ for every $n<\omega$. For $g \in F$ define $g^{\prime} \in \omega^{\omega}$ in the following way:

$$
g^{\prime}: \frac{g(1)}{k_{1} \text { times }}+\frac{g(2)}{k_{2} \text { times }}+\frac{g(3)}{k_{3} \text { times }}
$$


Now apply (iii) to the family $\left\{g^{\prime}: g \in F\right\}$ and define $J_{n}=I_{j}$ of minimal power from the $k_{n}$ th block for $n<\omega$. We have

$$
\infty>\sum_{n=1}^{\infty} \frac{\left|I_{n}\right|}{f^{\prime}(n)} \geqslant \sum_{n=1}^{\infty} \frac{k_{n}\left|J_{n}\right|}{f(n)}=\sum_{n=1}^{\infty} \frac{\left|J_{n}\right|}{n^{2}} .
$$

Thus $\left|J_{n}\right|<n^{2}$ for almost every $n<\omega$ and $\forall g \in F \forall{ }^{\infty} n g(n) \in J_{n}$. This finishes the proof of the lemma.

Now we will prove that $A(m) \rightarrow$ (iii). By Theorem 4 we already know that $A(m) \rightarrow D$. Take any family $F \subset \omega^{\omega}, F \prec f$ such that $|F|<2^{\omega}$ and $\sum_{n=1} f(n)^{-1}<\infty$. Let $X=\prod_{n=1}^{\infty} f(n)$ and

$$
H_{g}=\left\{x \in X: \exists^{\infty} n x(n)=g(n)\right\} \quad \text { for } g \in X .
$$

Denote by $\mu$ the standard product measure on $X$.

$$
\begin{aligned}
\mu\left(H_{g}\right) & =\mu\left(\bigcap_{n} \bigcup_{m>n}\{x \in X: x(m)=g(m)\}\right) \\
& \leqslant \mu\left(\bigcup_{m>n}\{x \in X: x(m)=g(m)\}\right) \leqslant \sum_{m>n} f(m)^{-1} \rightarrow 0 \quad \text { as } n \rightarrow \infty .
\end{aligned}
$$

Thus $\mu\left(H_{g}\right)=0$ for $g \in X$. By $A(m)$ we can find a closed set, i.e. a tree $T$ such that the set of its branches [T] has positive measure and $[T] \cap H_{g}=\varnothing$ for $g \in F$. Denote $T(n)=\{x(n): x \in[T]\}$ and $T_{s}=\{t \in T: s \leqslant t\}$. We can assume that $\left[T_{s}\right]$ has positive measure for $s \in T$.

Claim. $\forall g \in F \exists s \in T \forall n>\operatorname{lh}(s) g(n) \notin T_{s}(n)$.

Proof. Assume that it is false for some $g \in F$. Then there is a branch $x \in[T]$ such that $\exists^{\infty} n x(n)=g(n)$. But this means that $[T] \cap H_{g} \neq \varnothing$, thus a contradiction.

Therefore for every $g \in F$ we have some subtree $T_{s}$ as in the claim: let us call them $T_{s_{1}}, T_{s_{2}}, T_{s_{3}}, \ldots$ Denote $I_{m}^{n}=T_{s_{n}}(m)$ for $n, m<\omega$. It is easy to see that

$$
\prod_{m=1}^{\infty} \frac{\left|I_{m}^{n}\right|}{f(m)}>0 \text { for all } n<\omega .
$$

By changing the first few $I_{m}^{n}$,s for every $n<\omega$ we can obtain $\left\{I_{m}^{n}: n, m<\omega\right\}$ (use the same name) such that

$$
\prod_{m=1}^{\infty} \frac{\left|I_{m}^{n}\right|}{f(m)}>1-2^{-n-1} \text { for } n<\omega .
$$

Now let $J_{m}=\bigcap_{n} I_{m}^{n}$ for $m<\omega$. Obviously $\Pi_{n}\left|J_{n}\right| / f(n)>0$. Let $I_{n}=f(n)-J_{n}$, $n<\omega$. It is not very hard to see that $\sum_{n=1}^{\infty}\left|I_{n}\right| / f(n)<\infty$ and $\forall g \in F \forall^{\infty} n g(n) \in I_{n}$. This finishes the proof of Theorem 5 .

THEOREM 6. $h D \rightarrow A(c)$.

Corollary. $A(m) \rightarrow A(c)$.

Proof. By Theorems 1 and 2 in order to prove Theorem 6 it is enough to show that (ii) implies

$$
\forall F<\omega^{\omega}\left[|F|<2^{\omega} \rightarrow \exists g \in \omega^{\omega} \forall f \in F \exists^{\infty} n f(n)=g(n)\right] .
$$


Take any family $F \subset \omega^{\omega},|F|<2^{\omega}$. We have to find $g \in \omega^{\omega}$ such that $\forall f \in F \exists^{\infty} n$ $f(n)=g(n)$. For $f \in F$ define $f^{\prime}$ in the following way:

$$
f^{\prime}(k)=f_{\left.\| k^{3},(k+1)^{3}\right), \quad k<\omega .}
$$

Applying (ii) to the family $\left\{f^{\prime}: f \in F\right\}$ we obtain $\left\{I_{n}: n<\omega\right\}$ such that $\left|I_{n}\right|<n^{2}$ for $n<\omega$ and $\forall f \in F \forall^{\infty} n f^{\prime}(n) \in I_{n}$. Notice that each $I_{n}$ consists of less than $n^{2}$ functions whose domains have power bigger than $n^{2}$ (because $(n+1)^{3}-n^{3}>n^{2}$ ). Thus it is easy to find the required function picking one value out of each function from $I_{n}$ for every $n<\omega$. This finishes the proof of Theorem 6 .

In fact we have proved

COROLlaRY. If unions of less than $\lambda$ null sets are null than unions of less than $\lambda$ meager sets are meager.

This was also proved later by J. Raisonnier and Y. Stern.

\section{REFERENCES}

1. Arnold Miller, Some properties of measure and category, Trans. Amer. Math. Soc. 266 (1981), 93-114.

2. , Additivity of measure implies dominating real, Proc. Amer. Math. Soc. (to appear).

00-785 Warsaw, Grottgera 12A-51, Poland 